\title{
Implementation of a nucleate boiling flux partitioning model for a CFD simulation of compact heat exchangers based on the local estimation of bulk properties
}

\author{
C. Paz, J. Porteiro, A. Copo \& A. Díaz \\ Escuela de Ingeniería Industrial, University of Vigo, Spain
}

\begin{abstract}
The widely used implementation of wall heat flux partitioning during subcooled flow nucleate boiling has a number of limitations when it is applied on a local cell basis as is done in CFD codes. The main limitations reported generally have their origin in the bulk formulation of the proposed submodels being transferred to a local balance at the cell near the wall whose volume and flow conditions strongly differ from those employed in the experimental calibration of the model imposed. Several models developed for nucleate boiling are based on a so-called wall heat flux partitioning strategy that consists of a modification of the convective heat transfer law at the wall to include evaporation. However, it is known that the local movement of the subcooled liquid to replace the volume of vapour represents an enormous contribution to heat flux enhancement, a process that is known as quenching. Therefore, when a $3 \mathrm{D}$, local formulation of a wall heat flux partitioning boiling submodel is implemented at the cell scale in a CFD code, it must take into account that most of the available correlations and the physical phenomenon itself are strongly connected with regions of the fluid that are quite distant from the wall, normally around $0.5 \mathrm{~mm}$. The numerical structure of CFD codes is not suitable for a non-local formulation. Therefore, this study attempts to address these limitations to implement a flux partitioning boiling model at the local cell scale but employs distant properties of the fluid for bulk conditions and heat exchange. This method has been validated in several geometries, allowing parallelisation and full grid size and shape versatility. The results obtained when applied to an EGR cooler indicate an important improvement with respect to other models, and the results obtained are more
\end{abstract}


realistic. Moreover, it could also be used in any physical model that requires the value of certain properties at some reference distance from the wall. Keywords: nucleate boiling, Chen's model, CFD, local properties.

\section{Introduction}

During several heat transfer processes when heat is being rejected, the coolant fluid used may locally boil at regions where the wall temperature is higher than the saturation temperature at the system's pressure. During the first stage of boiling, known as nucleate boiling, the heat transfer is increased because of the agitation caused by the small bubbles that form over the heated wall and subsequently move away from the wall when buoyancy forces go beyond superficial forces. In addition, the heat transfer rate is increased because of the capability of those bubbles to transport heat to regions far away from the wall as latent heat. As a result, non-boiling codes over-predict the wall temperature because this increment of heat transferred is not taken into account, leading to non-physical temperatures of the fluid at the wall, incorrect predictions of system's performance, thermal stresses or any other aspect being studied.

In the last few decades, the study of heat transfer in boiling processes has become increasingly important in a number of industrial sectors such as the nuclear and automobile industries. To predict this heat transfer more accurately, several boiling models have been developed to introduce the physics of this process into CFD codes. However, most of boiling models in commercial CFD codes have been created for applications in the nuclear industry, where a higher pressure is employed and, hence, the volumetric expansion of the vapour bubbles is relatively low compared with normal automotive applications. This difference, together with the fact that those submodels are based on specific experimental correlations, causes boiling subroutines developed for the nuclear industry to usually be unreliable and numerically unstable when applied at low pressure.

Nevertheless, there are a number of submodels suitable for CFD implementation at low-pressure, but they still require empirical correlations that are based on the bulk properties of the fluid obtained in an experimental test section.

In contrast, the numerical structure of CFD codes as well as its power lies in the local formulation of the mass, momentum and energy balances (among others). This makes the method efficient in solving the fluid field in complex geometries and under complex boundary conditions normally present in the industry. Special mention should be made regarding the transition from the turbulent core of the flow towards the laminar boundary layer at the wall, where a very small discretisation is the only way of ensuring accuracy. Therefore, the multi-scale phenomena generated by boiling are not straightforward in a CFD code, where local properties at the wall may strongly differ from the properties at the distance of influence of the boiling phenomena. Taking into account that the departure of a bubble injects the same volume of cold liquid towards the wall in a process known as quenching, it can be easily established that the bubble scale is relevant in this process. In fact, this contribution to heat transfer is the greatest 
during nucleate boiling as demonstrated by Kurul and Podowski [1]. As a reference, and based on experimental studies performed by Del Valle and Kenning [2] and Tulobinsky and Konstanczuk [3], in this study, this scale is set to $0.5 \mathrm{~mm}$. Hence, the proper implementation of the boiling model will require that the properties of the liquid at the bubble's scale are available for each cell at the wall.

Therefore, in this study, an accurate and computationally efficient method to retrieve properties far from the wall, without exhibiting any limitations because of serial or parallel processes, mesh size or cell type, or case geometry is presented. This procedure has been applied to a boiling model, but it could also be used in other physical models that require properties at a distance from the wall.

\section{Modelling}

For several physical models in the literature, the bulk properties are some of the parameters taken into account. Typically, those models are based on empirical correlations that, in turn, are based on global formulations of the bulk properties. However, because the objective of a numerical calculation is to obtain the details of the case, a global approach of any model appears to be contrary to the purpose of CFD.

In addition to the global formulation of the bulk properties, where they are constant in the entire domain of the case, an alternative to obtaining the local properties has been proposed by Krepper et al. [4] that consists of a mathematical reconstruction of the law of the wall. However, the solution given by this proposal is mesh-dependent and is only valid for fully-developed flows, which represents a strong constraint regarding typical automotive designs, where extreme compactness constrains the lengths required for the full development of the flow.

To address all these limitations, a methodology to obtain the local properties has been developed, based on the CFD solution of the case.

\subsection{Formulation of the model}

The physical model chosen for the CFD implementation is a modified version of Chen's model [5], which is a boiling model widely used in the automotive industry because of its substantial simplicity and generality.

This model consists of the partitioning of the wall heat flux into two terms: one related to forced convection heat flux, multiplied by a factor $F$, and the other related to the enhancement of heat flux caused by nucleate boiling, multiplied by the factors $S_{f c}$ and $S_{s u b}$. Therefore, the wall heat flux is expressed as

$$
q_{w}=F q_{f c}+S_{f c} S_{s u b} q_{n b},
$$

where $q_{f c}$ stands for the forced-convection heat flux and $q_{n b}$ represents the nucleate boiling heat flux, whose equations are as follows: 


$$
\begin{gathered}
q_{f c}=h_{f c}\left(T_{w}-T_{b}\right) \\
q_{n b}=h_{n b}\left(T_{w}-T_{s a t}\right) .
\end{gathered}
$$

In the original formulation of Chen's model, $h_{f c}$ is the forced-convection heat transfer coefficient obtained by any of the available correlations such as those given by Dittus and Boelter [6], Gnielinski [7], or Petukhov [8]. However, in a CFD framework, equation 2 is replaced by the single-phase heat transfer already obtained by CFD. The nucleate boiling heat transfer coefficient $h_{n b}$ is obtained using the correlation of Forster and Zuber [9]:

$$
\begin{gathered}
h_{n b}=0.00122 \frac{k_{l}^{0.79} c_{p l}^{0.45} \rho_{l}^{0.49}}{\sigma^{0.5} \mu_{l}^{0.29} h_{l v}^{0.24} \rho_{v}^{0.24}}\left(T_{w}-T_{\text {sat }}\right)^{0.24}\left[P_{\text {sat }}\left(T_{w}\right)-\right. \\
\left.P_{\text {sat }}\left(T_{\text {sat }}\right)\right]^{0.75}
\end{gathered}
$$

where $F$ accounts for the convective heat transfer enhancement caused by liquid agitation when a bubble develops and lifts off from the wall. According to Chen's original formulation, the $F$ factor is related to the inverse of the Martinelli number

$$
\left(\frac{1}{X_{t t}}\right)=\left(\frac{\zeta}{1-\zeta_{v}}\right)^{0.9}\left(\frac{\rho_{l}}{\rho_{v}}\right)^{0.5}\left(\frac{\mu_{v}}{\mu_{l}}\right)^{0.1}
$$

where $\zeta_{v}$ is the vapour mass fraction. Butterworth [10] developed an analytical correlation to obtain the value of the $F$ factor:

$$
\begin{cases}F=2.35\left(\frac{1}{x_{t t}}+0.213\right)^{0.736} & \text { si } \zeta_{v}>0.1 \\ F=1 & \text { si } \zeta_{v} \leq 0.1\end{cases}
$$

In the boiling model proposed in this paper, it is assumed that the mass fraction is small enough to assume that the $F$ factor is equal to one.

The subcooled boiling factor $S_{s u b}$ and the suppression forced convection $S_{f c}$ factor are obtained using the Steiner et al. [11] and Butterworth [10] correlation, respectively:

$$
\begin{gathered}
S_{f c}=\frac{1}{1+2.53 \cdot 10^{-6}\left(R_{l} F^{1.25}\right)^{1.17}} \\
S_{s u b}=\frac{T_{w}-T_{s a t}}{T_{w}-T_{b}} .
\end{gathered}
$$

\subsection{Numerical implementation}

The software used in this study was Ansys-Fluent, which allows the implementation of user-defined functions (UDF) written in the $\mathrm{C}$ programing 
language [12]. The full code of the boiling model is divided in two steps: The first step consists of modifying the wall heat transfer law based on the Chen-type model. The second step consists of determining the local-bulk properties, which, though seemingly contradictory, means the bulk properties of the liquid at a certain distance from every specific position at the wall, i.e., the properties of the liquid layer that may have influence on each specific position of the wall in the case of boiling.

The proposed nucleate boiling model has several parameters that can be considered as local-bulk properties. These properties are $k, C_{p}, \rho_{l}$, and $\mu$ in Forster and Zuber's nucleate boiling convection coefficient; $T_{b}$ in $q_{f c}$; and the Reynolds number in $S_{s u b}$.

To obtain the value of the local properties, a searching algorithm based on the geometry of the case has been developed. This algorithm creates an assignation between each face centroid of a wall and a cell centroid from the fluid domain adjacent to this boiling wall. Each face centroid searches for the cell centroid in the direction normal to its own face at a reference distance, which can been established as an input of the algorithm, that was set to $0.5 \mathrm{~mm}$ in this study because this is representative of the average bubble diameter that approximately represents the distance from which liquid will be injected towards the wall because of quenching.

If at the established distance and within a small tolerance, also set as an input, a cell centroid is found, those cell properties are assigned as bulk properties. Otherwise, the tolerance margins are increased until a cell centroid is found.

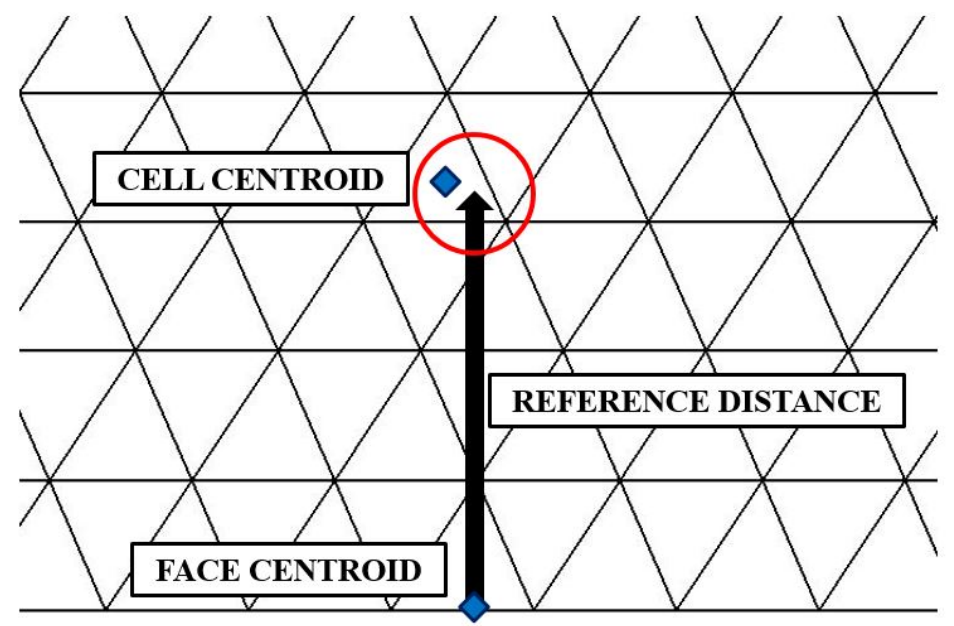

Figure 1: Schematic of the proposed method.

This method has proven to be accurate in structured and non-structured mesh, serial and parallel calculations and using any type of cell. In addition, the 
computational time required by this algorithm is very small compared to the computational time of the problem being solved.

Because this method is based on the coordinates of the face and cell centroids, no restrictions have been found for its application to any type of model.

\section{Experimental setup}

The predictive capability of the proposed model was evaluated by comparing its predictions with the experimental data obtained in a test facility that is able to record both temperatures and heat fluxes across a heated wall. The experiments performed were designed to investigate subcooled convective boiling at typical working conditions for coolant systems of internal combustion engines.

According to the literature, there are several other experimental setups with different geometries; although in those cases, where the field of application is the automotive industry, a small, flat plate is normally used. For these experiments, a $25 \mathrm{~mm}$ high by $20 \mathrm{~mm}$ wide rectangular section was chosen, similar to the experiments of Robinson et al. [13], Steiner et al. [11], Klausner et al. [14], or Maurus et al. [15]. An up to $2 \mathrm{MW} / \mathrm{m}^{2}$ heat flux was introduced into the channel by a small boiling surface that is in contact with an electrically heated copper block that is located at the bottom of the duct. Six thermocouples were distributed in the block to perform estimations using Fourier Law. Deionised water was used as the working fluid.

Windows made of glass were embedded in the top as well as the lateral walls to make the test duct visible. Figure 2 gives a schematic view of the test stand used for the experiments.

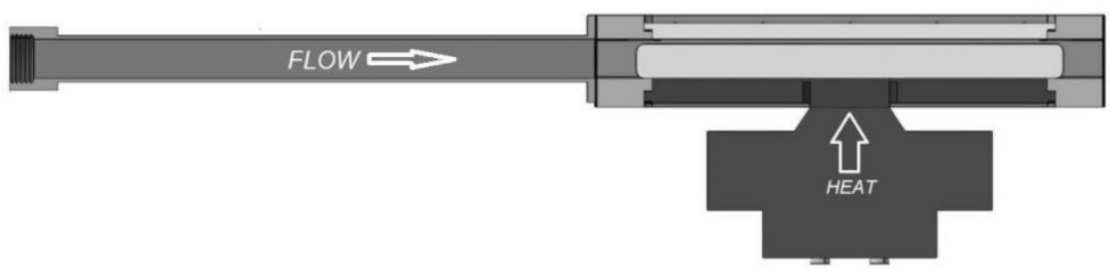

Figure 2: Experimental setup.

A PTFE insulator surrounds the top of the copper block. The low thermal conductivity of this material guarantees maximum heat transfer to the working fluid and minimum heat loss to the duct structure, which allows a proper prediction of the thermal flux towards the heating element.

\section{Results}

Using the test stand described in section 3, boiling curves were obtained in three experimental cases for different system pressures, inlet velocities, and inlet temperatures. The conditions studied are listed in Table 1. 
Table 1: Cases analysed.

\begin{tabular}{|c|c|c|c|}
\cline { 2 - 4 } \multicolumn{1}{c|}{} & Inlet velocity $[\mathrm{m} / \mathbf{s}]$ & Inlet temperature $\left[{ }^{\circ} \mathbf{C}\right]$ & System pressure $[\mathrm{kPa}]$ \\
\hline CASE 1 & 0.25 & 90 & 125 \\
\hline CASE 2 & 0.5 & 85 & 150 \\
\hline CASE 3 & 0.75 & 80 & 175 \\
\hline
\end{tabular}

The experimental boiling curves were contrasted with the results obtained in the analogue CFD simulations, showing good agreement in every case and in both partially- and fully-developed boiling regimes. Figure 3 through Figure 5 compare the experimental and predicted boiling curves.

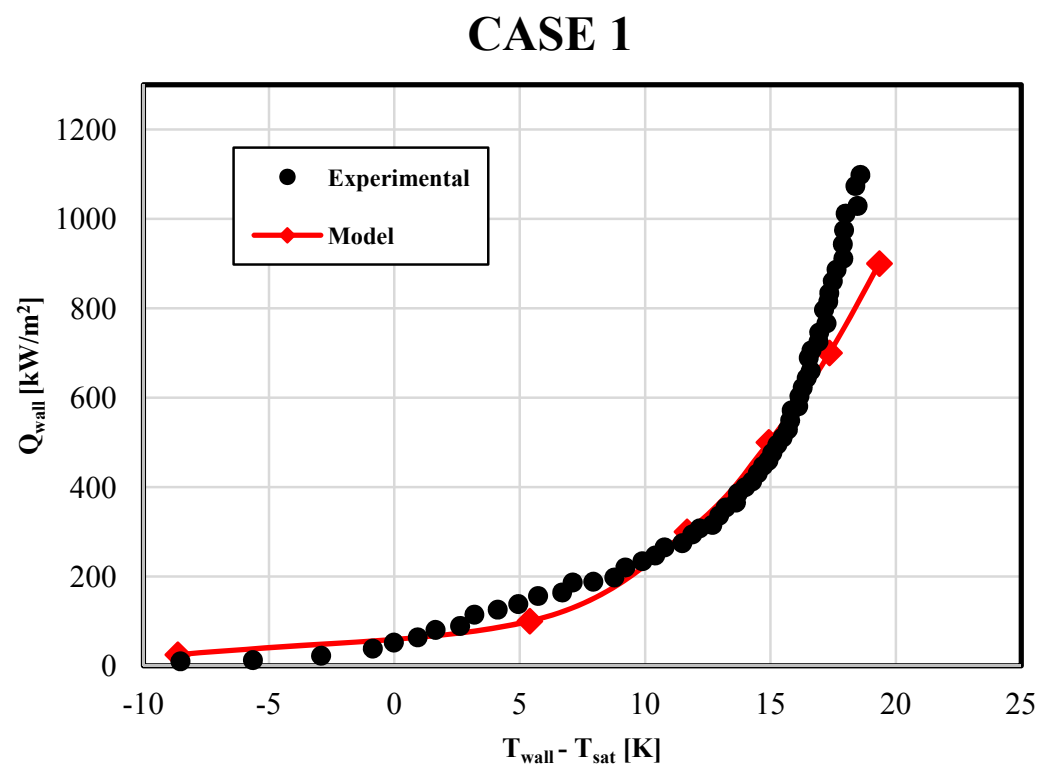

Figure 3: $\quad$ Boiling curve for Case 1.

\section{Conclusions}

In this study, a Chen-type nucleate boiling model was developed to calculate the wall heat flux in subcooled boiling flow via CFD. To take into account the quenching contribution to the heat flux, a method to determine the bulk properties at a distance far from the wall was developed. The proposed model is in good agreement with the experimental data, in both the partially-developed 
CASE 2

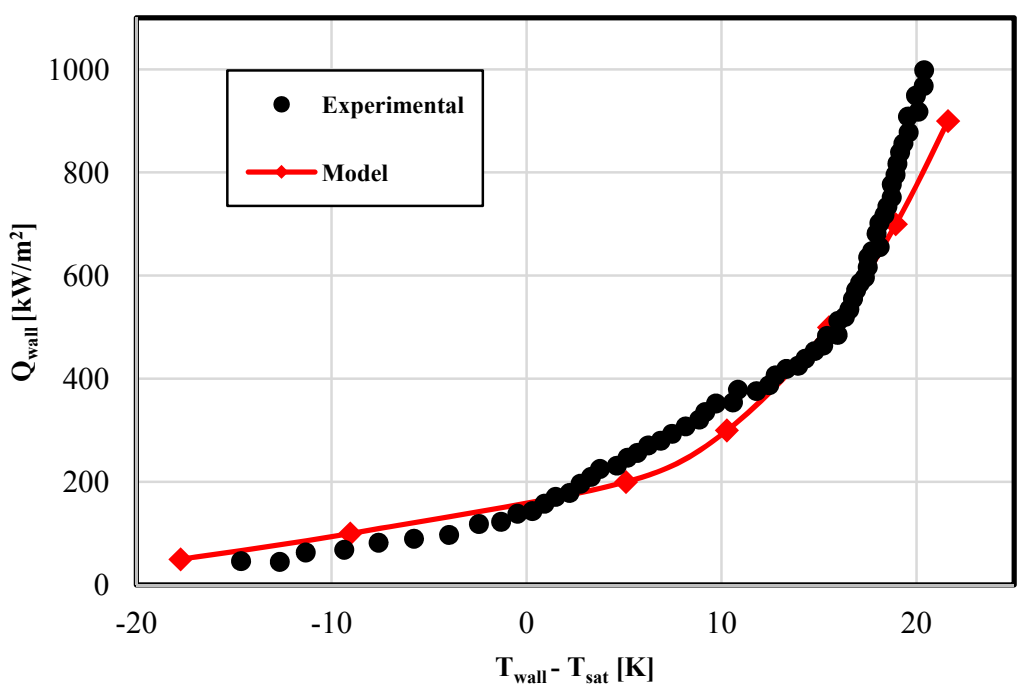

Figure 4: Boiling curve for Case 2.

\section{CASE 3}

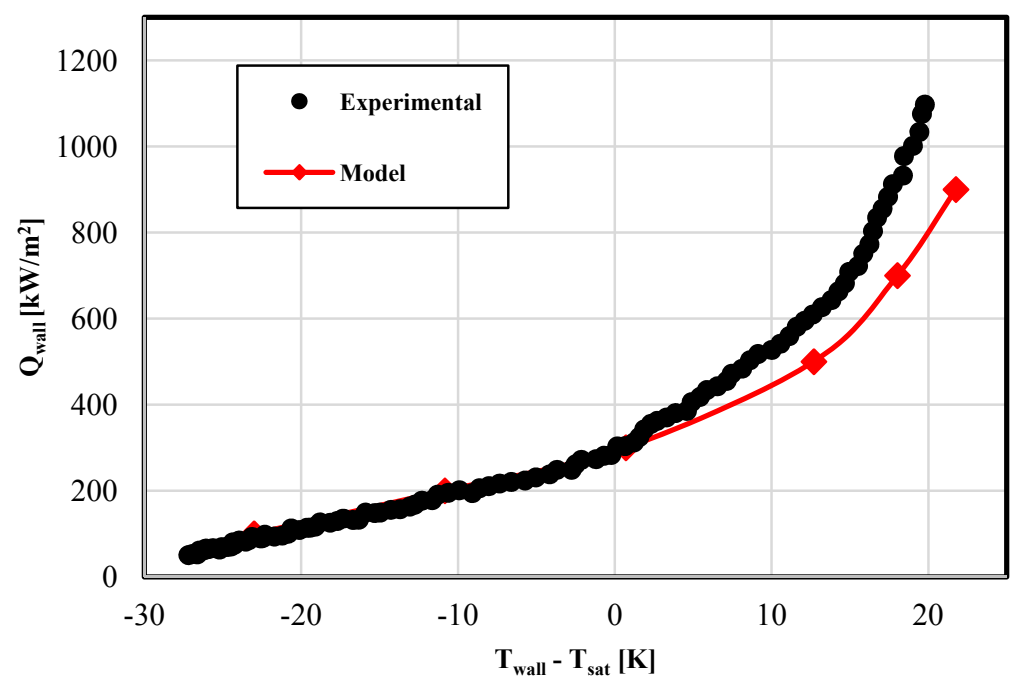

Figure 5: Boiling curve for Case 3. 
boiling (PDB) regime and the fully-developed boiling (FDB), in different inlet velocity ranges, inlet temperatures and system pressures.

The developed methodology to obtain properties far from the wall could also be applied to any other physical model demanding certain values of properties at some distance far from the wall.

\section{Acknowledgement}

We gratefully acknowledge BorgWarner Emissions Systems for providing material and technical support.

\section{Nomenclature}

$\begin{array}{ll}D_{h} & \text { hydraulic diameter }[\mathrm{m}] \\ F & \text { correction factor }[-] \\ h & \text { heat transfer coefficient }\left[\mathrm{W} / \mathrm{m}^{2} \mathrm{~K}\right] \\ h_{l v} & \text { latent heat }[\mathrm{J} / \mathrm{kg}] \\ k & \text { thermal conductivity }[\mathrm{W} / \mathrm{m} \mathrm{K}] \\ P & \text { pressure }\left[\mathrm{N} / \mathrm{m}^{2}\right] \\ q & \text { specific heat transfer rate }\left[\mathrm{W} / \mathrm{m}^{2}\right] \\ R e & \text { bulk flow Reynolds number }[-] \\ S & \text { suppression factor }[-] \\ T & \text { temperature }\left[{ }^{\circ} \mathrm{C}\right] \\ Q & \text { flow rate }\left[\mathrm{m}^{3} / \mathrm{s}\right] \\ X_{t t} & \text { Martinelli number }[-]\end{array}$

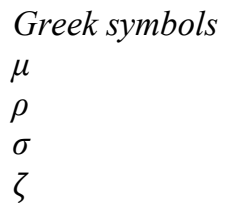

dynamic viscosity $[\mathrm{kg} / \mathrm{m} \mathrm{s}]$ density $\left[\mathrm{kg} / \mathrm{m}^{3}\right]$ surface tension $\left[\mathrm{kg} / \mathrm{s}^{2}\right]$ mass fraction [-]

$\begin{array}{ll}\text { Subscripts } & \\ b & \text { bulk } \\ f_{c} & \text { forced convection } \\ l & \text { liquid phase } \\ n b & \text { nucleate boiling } \\ \text { sat } & \text { saturation } \\ \text { sub } & \text { subcooling } \\ v & \text { vapour phase } \\ w & \text { wall }\end{array}$




\section{References}

[1] N. Kurul and M. Z. Podowski, "On the modeling of multidimensional effects in boiling channels," in Proceedings of the 27th National Heat Transfer Conference, Minneapolis, USA, 1991.

[2] V. H. Del Valle and D. B. R. Kenning, "Subcooled flow boiling at high heat flux," International Journal of Heat and Mass Transfer, no. 28(10), pp. 1907-1920, 1985.

[3] V. I. Tolubinsky and D. M. Kostanczuk, "Vapour Bubbles Growth Rate and Heat Transfer Intensity at Subcooled Water Boiling," Proc. $4^{\text {th }}$ International Heat Transfer Conference, vol. 5, $\mathrm{n}^{\circ} 1970$.

[4] E. Krepper, B. Koncar and Y. Egorov, "CFD modelling of subcooled boiling - Concept, validation and application to fuel assembly design," Nuclear Engineering and Design, no. 237, pp. 716-731, 2007.

[5] J. C. Chen, "Correlation for Boiling Heat Transfer to Saturated Fluids in Convective Flow," Industrial \& Engineering Chemistry Process Design and Development, vol. 5, pp. 322-329, July 1966.

[6] F. W. Dittus and L. M. K. Boelter, "Heat transfer in automobile radiators of the tubular type," International Communications in Heat and Mass Transfer, no. 12(1), pp. 3-22, 1985.

[7] V. Gnielinski, "New equation for heat and mass transfer in turbulent pipe and channel flow," International Chemical Engineering, no. 16(2), pp. 359-368, 1976.

[8] B. S. Petukhov, Heat Transfer and Friction in Turbulent Pipe flow with Variable Physical Properties, New York: Academic Press, 1970.

[9] H. K. Forster and N. Zuber, "Dynamics of vapor bubbles and boiling heat transfer," AIChE Journal, vol. 1, pp. 531-535, 1955.

[10] D. Butterworth, "The correlation of cross flow pressure drop data by means of a permeability concept," UKAEA Report AERE-R9435, 1979.

[11] H. Steiner, A. Kobor and L. Gebhard, "A wall heat transfer model for subcooled boiling flow," International Journal of Heat and Mass Transfer, no. 48, pp. 4161-4173, 2005.

[12] Ansys, Fluent 14. User's Guide.

[13] K. Robinson, J. G. Hawley and N. A. F. Campbell, "Experimental and modelling aspects of flow boiling heat transfer for application to internal combustion engines," Proceedings of the Institution of Mechanical Engineers, no. 217(10), pp. 877-889, 2003.

[14] J. F. Klausner, L. Z. Zeng and D. M. Bernhard, "Development of a film thickness probe using capacitance for asymmetrical flow with heat addition," Review of Scientific Instruments, no. 63, pp. 3147-3152, 1992.

[15] R. Maurus, V. Ilchenko and T. Sattelmayer, "Study of the bubble characteristics and the local void fraction in subcooled flow boiling using digital imaging and analysing techniques," Experimental Thermal and Fluid Science, no. 26, pp. 147-155, 2002. 